\title{
Modulation de l'expression du pouvoir pathogène de Botrytis fabae Sard. et de Botrytis cinerea Pers. par des bactéries du phylloplan de Vicia faba L.
}

\author{
Régis VEDIE \& Michel LE NORMAND
}

E.N.S.A. - I.N.R.A., Laboratoire de Botanique et Pathologie végétale, 65, Rue de Saint-Brieuc, F 3.5042 Rennes Ceder

La formation et le développement de lésions nécrotiques par Botrytis fabae et $B$. cinerea sur feuilles détachées de Vicia faba sont inhibés par la présence de sulfate ferrique dans la goutte d'inoculum. Le sulfate ferrique n'a pas d'effet sur la germination des conidies de Botrytis sur lames de verre, mais entraîne une forte stimulation de la croissance des tubes germinatifs. L'inhibition observée sur les feuilles détachées ne s'explique pas par la production de phytoalexines ni par la stimulation des réactions de défense de la plante. Par contre, les modifications relevées dans la composition de la microflore du phylloplan paraissent essentielles. Deux bactéries prédominantes suivant les conditions ont été isolées. Pseudomonas fluorescens est isolé dans les gouttes d'inoculum donnant des lésions, alors qu'en présence de sulfate ferrique - et donc en l'absence de lésions - c'est Erwinia herbicola qui devient majoritaire. Sur milieu de KING B, Pseudomonas exerce une antibiose vis-à-vis d'Erwinia. D'autre part, Erwinia inhibe la germination des conidies ainsi que la croissance des tubes germinatifs des Botrytis lors de tests in vitro. Mais on note également que l'inhibition des lésions due au sulfate de fer est presque totalement annulée par l'addition de Pseudomonas à l'inoculum.

Le mode d'action du fer est discuté en terme d'avantage sélectif procuré à $E$. herbicola par rapport à $P$. fluorescens.

Mots clés additionnels : Fer, microflore, antibiose, sidérophores, Pseudomonas fluorescens, Erwinia herbicola.

Modulation of the pathogenicity of Botrytis fabae Sard. and Botrytis cinerea Pers. by bacteria in the phylloplane of Vicia faba $L$.

Initiation and development of necrotic lesions by Botrytis fabae and B. cinerea on detached leaves of Vicia faba was inhibited by ferric sulfate in inoculum drops. Ferric sulfate had no effect on conidial germination of the two species on glass slides but a high stimulation of germ-tube growth was observed in the presence of iron. The inhibition observed on detached leaves could not be explained by phytoalexin production or by the stimulation of defence reactions in the plant. However, modifications in the microbial composition of the phyllophane flora seemed to play an important role. Two predominant bacteria were isolated under different conditions. Pseudomonas fluorescens was obtained from inoculum drops over developing necrotic lesions while Erwinia herbicola became dominant in ferric sulfate drops without necrotic lesions.

On KING B medium, the $P$. fluorescens strain inhibited growth of $E$. herbicola, while in turn Erwinia inhibited conidial germination and germ tube growth of both Botrytis sp. on glass slides. Finally the inhibition of lesion development by ferric sulfate was almost completely suppressed by addition of the Pseudomonas strain to the inoculum.

The mode of action of iron is discussed in terms of the selective advantage given to $E$. herbicola against $P$. fluorescens.

Additional key words : Iron, microflora, antibiosis, siderophores, Pseudomonas fluorescens, Erwinia herbicola.

\section{INTRODUCTION}

L'importance du fer dans les relations s'établissant entre divers micro-organismes pathogènes (bactéries, champignons) et leurs hôtes a été soulignée dans de nombreuses études (WEINBERG, 1966, 1978). On a même montré que la virulence de Vibrio anguillarum Bergman (CROSA, 1980) est liée à la présence d'un plasmide codant pour un chélateur du fer.

Dans des milieux très pauvres en ions ferriques libres 
et solubles, des bactéries et certains champignons sécrètent des sidérophores (LANKFORD, 1973), composés ayant une forte affinité pour le fer et assurant l'alimentation en fer de ces micro-organismes.

Mais l'activité des sidérophores ne se limite pas à ce seul effet nutritif ; ils peuvent, par leur plus ou moins forte affinité pour le fer ou par leur spécificité plus ou moins étroite pour le micro-organisme qui les produit, moduler la compétitivité de ce micro-organisme.

Par exemple, certains Pseudomonas du sol seraient responsables de la limitation d'autres rhizobactéries, parasites mineurs, grâce à une activité antagoniste due à la production de tels sidérophores (SCHROTH \& HANCOCK, 1982). Le fer serait ainsi mobilisé au profit des Pseudomonas et peut-être des plantes, entraînant de notables augmentations du rendement des plantes cultivées.

A l'inverse, à la surface des organes aériens des plantes, des bactéries productrices de sidérophores semblent plutôt augmenter les dégâts dus à certains agents pathogènes. Ainsi des Pseudomonas saprophytes, producteurs de sidérophores, stimulent la germination des conidies de Colletotrichum musae (Berk \& Curt) Arx. (Mc CRACKEn \& SWinburne, 1979). De plus, celles-ci ont une agressivité d'autant plus forte que le milieu de culture d'origine est plus pauvre en fer (BROWN \& SwINBURNE, 1981), ce qui pourrait être lié à la régulation endogène par le fer des sidérophores propres du champignon. Ces sidérophores, permettant une concurrence efficace pour l'alimentation en fer, favoriseraient ainsi le pouvoir pathogène du champignon.

De même, dans la relation Botrytis cinerea Pers. Vicia faba, BROWN \& SWINBURNE (1982) ont montré que les chélateurs du fer, EDTA (Acide éthylène diamine tétraacétique) et DHBA (Acide dihydroxybenzoïque), augmentent l'agressivité des conidies de $B$. cinerea. L'addition de sulfate ferrique $\left(\mathrm{Fe}_{2}\left(\mathrm{SO}_{4}\right)_{3}\right)$ à l'inoculum traité par ces chélateurs réduit cette agressivité au niveau antérieur.

Ces divers résultats nous ont incités à définir le rôle éventuel du fer dans l'interaction Botrytis - Vicia $f a b a$, actuellement étudiée dans le laboratoire. Les investigations entreprises nous ont très vite conduits à nous interroger sur l'importance possible de certaines bactéries du phylloplan dans la régulation de l'expression du pouvoir pathogène de $B$. fabae Sard. et de B. cinerea.

\section{MATÉRIELS ET MÉTHODES}

\section{A. Plantes et agents pathogènes}

Les graines des lignées de féverole 29E, LCF, S45, fournies par M. BERTHELEM du Laboratoire d'Amélioration de la féverole de l'I.N.R.A. (Le Rheu-Rennes), sont semées dans un mélange de vermiculite et de tourbe (1 pour 2). Ces lignées sont choisies pour leurs sensibilités différentes vis-à-vis de $B$. fabae. Les feuilles sont prélevées lorsque les plantes sont au stade 4 feuilles bifoliolées.

Les souches B. fabae (AX) et $B$. cinerea (GZ), isolées au champ en Egypte par ABOU-ZEID (1978), sont cultivées sur milieu malt-agar enrichi en saccharose $(3 \mathrm{p} .100)$ et nitrate de sodium ( 3 p. 100) de façon à obtenir une bonne sporulation (LEACH \& MOORE, 1966).

\section{B. Inoculum et contamination}

Les suspensions de spores sont préparées à partir de cultures âgées de $12 \mathrm{j}$. La surface de la colonie sporulée est vigoureusement agitée dans de l'eau distillée. Une filtration sur coton de verre permet d'éliminer les fragments mycéliens grossiers. La concentration finale de la suspension est ajustée à $5 \times 10^{3}$ conidies $/ \mathrm{ml}$.

Les spores sont déposées en gouttes calibrées de $20 \mu \mathrm{l}$ sur la face inférieure des feuilles détachées, placées en chambre humide et incubées à l'obscurité à $25^{\circ} \mathrm{C}$.

La notation des nécroses est faite, après différentes durées d'incubation, selon l'échelle de MANSFIELD \& DEVERALL (1974) adaptée à notre matériel par ABOUZEID (1978). Cette échelle comprend 10 notes : de 0 à 5 nous notons l'apparition et le développement des taches nécrotiques sous la goutte d'inoculum et de 6 à 9 l'évolution de la nécrose à l'extérieur de la goutte. Les résultats sont exprimés en pourcentage de la note maximale.

\section{Isolement des bactéries}

Les isolements sont effectués à partir des suspensions de spores ayant séjourné sur les folioles pendant $24 \mathrm{~h}$ en présence soit d'eau, soit d'EDTA, soit de fer. Ces gouttes sont étalées sur milieu KING B. (KING et al., 1954) sur lequel Botrytis ne peut se développer tandis que les bactéries forment des colonies.

Pour chaque traitement, 10 isolements sont réalisés et l'expérience est répétée 3 fois.

\section{Epreuve de germination des conidies}

Lors des différents tests entrepris dans cette étude, gouttes sont étalées sur milieu KING B (KING et al., avec un volume égal de différentes solutions de façon à obtenir les molarités finales indiquées :

- solutions d'EDTA à $10^{-4} \mathrm{M}$;

- solution de $\mathrm{Fe}_{2}\left(\mathrm{SO}_{4}\right)_{3}$ à $10^{-4} \mathrm{M}$;

- un mélange de ces 2 substances à la concentration finale de $10^{-4} \mathrm{M}$.

Pour étudier l'effet des bactéries, on apporte aux mélanges précédents un volume égal de suspension bactérienne $\left(10^{6}\right.$ germes $\left./ \mathrm{ml}\right)$. Les mélanges obtenus sont déposés soit sur la face inférieure des folioles (pour l'évaluation de l'effet du traitement sur l'expression du pouvoir pathogène) soit sur lames de verre (pour l'évaluation de l'effet sur la germination et la croissance des tubes germinatifs in vitro). Pour chaque traitement, 4 dépôts sont effectués sur 5 folioles différentes et l'expérience est répétée 3 fois. Pour les tests sur lames de verre, le pourcentage de germination est estimé sur 500 spores et la croissance des tubes germinatifs par la mesure de leur longueur sur 100 spores germées.

\section{E. Recherches de phytoalexines}

Ces recherches sont faites à 2 niveaux :

- Dans les gouttes déposées sur les feuilles (var. S45) contenant des spores de $B$. fabae (AX) en présence 
d'eau ou de sulfate ferrique, après une incubation de 24 ou $72 \mathrm{~h}$. Ces solutions recueillies sont filtrées sur filtres Sartorius $0,45 \mu \mathrm{m}$.

- Dans les diffusats des feuilles ayant porté ces gouttes; les feuilles sont infiltrées sous vide par une solution d'alcool à 80 p. 100 (KEEN, 1978), placées sur une grille dans un tube à centrifuger (KLEMENT, 1965) et le liquide ou diffusat recueilli par centrifugation au fond du tube est débarrassé de l'alcool par évaporation sous-vide à $40^{\circ} \mathrm{C}$.

Le schéma d'extraction des phytoalexines dans les solutions ainsi obtenues est inspiré des travaux de HARGREAVES et al. (1977). Les solutions obtenues, par les 2 méthodes, sont acidifiées à $\mathrm{pH}=5$ par $\mathrm{H}_{3} \mathrm{PO}_{4}$, puis traitées 3 fois au diéthyl oxyde. Les fractions solubles dans le diéthyl oxyde sont regroupées, évaporées, puis reprises dans du méthanol au volume initial.

Les différentes solutions obtenues sont soumises à une chromatographie sur couche mince (gel de silice 60 de Merck), développée dans le système chloroforme éther de pétrole $(2: 1)$. La recherche de zones inhibitrices sur le chromatogramme est faite par pulvérisation d'une suspension de spores très dense de Cladosporium $s p$. préparée dans un milieu nutritif de Czapek liquide.

\section{RÉSULTATS}

\section{A. Modification du pouvoir pathogène des Botrytis de Vicia faba par l'apport de fer}

\section{Mise en évidence du phénomène}

Après 24 h d'incubation, tant pour $B$. fabae que pour $B$. cinerea, on note une inhibition importante de l'apparition des taches nécrotiques dans les gouttes d'inoculum contenant du sulfate ferrique (fig. 1).

Avec l'EDTA, l'inhibition est faible sur la variété 29E, pour la souche $A X$, et non significative sur la variété $\mathrm{S} 45$, pour la souche GZ. Dans le mélange EDTA plus $\mathrm{Fe}_{2}\left(\mathrm{SO}_{4}\right)_{3}$, le niveau des symptômes est comparable à celui obtenu dans l'eau distillée.

Après $48 \mathrm{~h}$ d'incubation, la tendance reste la même. Il y a cependant une augmentation de la maladie pour l'ensemble des traitements.

Ces premiers résultats font donc apparaître une inhibition significative du pouvoir pathogène des Botrytis sur feuilles détachées de $V$. faba en présence de sulfate ferrique $\left(10^{-4} \mathrm{M}\right)$.

\section{Etude du phénomène en fonction des doses d'inocu- lum et des concentrations en fer}

Nous avons précisé cette action inhibitrice en utilisant plusieurs concentrations de fer et une série de doses d'inoculum (spores de B. fabae) (fig. 2). Pour des concentrations en fer supérieures ou égales à $5 \cdot 10^{-4} \mathrm{M}$, l'inhibition est effective sur toutes les doses d'inoculum. Pour la plus faible concentration en fer dans la goutte d'inoculum $\left(5.10^{-5} \mathrm{M}\right)$, l'inhibition n'est significative que sur la plus faible dose d'inoculum $\left(10^{3}\right.$ spores $/ \mathrm{ml}$ ) et pour une durée d'incubation de $24 \mathrm{~h}$. Cette dose se rapproche d'un inoculum naturel et les concentrations en fer qui se révèlent inhibitrices pourraient correspondre à celles utilisées pour des fertilisations foliaires en oligo-éléments.

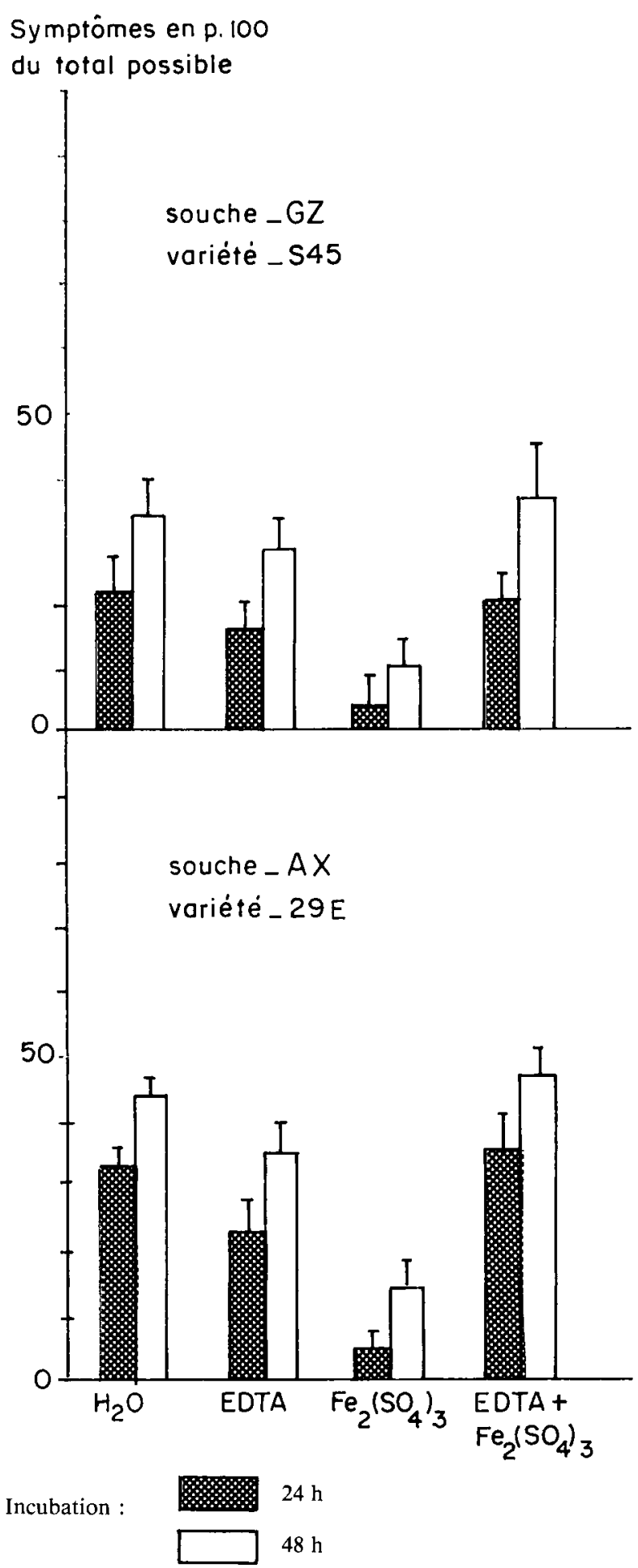

Figure 1

Effets de divers traitements sur le pouvoir pathogène de Botrytis fabae $(A X)$ et de $\mathrm{B}$. cinerea $(G Z)$ sur feuilles détachées de Vicia faba I limites de confiance $(95 \mathrm{p}, 100)$.

Effects of various treatments on the pathogenicity of Botrytis fabae $(A X)$ and $\mathrm{B}$. cinerea (GZ) on detached leaves of Vicia faba I Confidence limits $(95 \%)$.

\section{B. Action du fer et de l'EDTA sur le parasite et sur la plante hôte}

\section{Au niveau du parasite}

Les produits testés n'ont montré aucune action significative sur la germination des spores de Botrytis. Par 


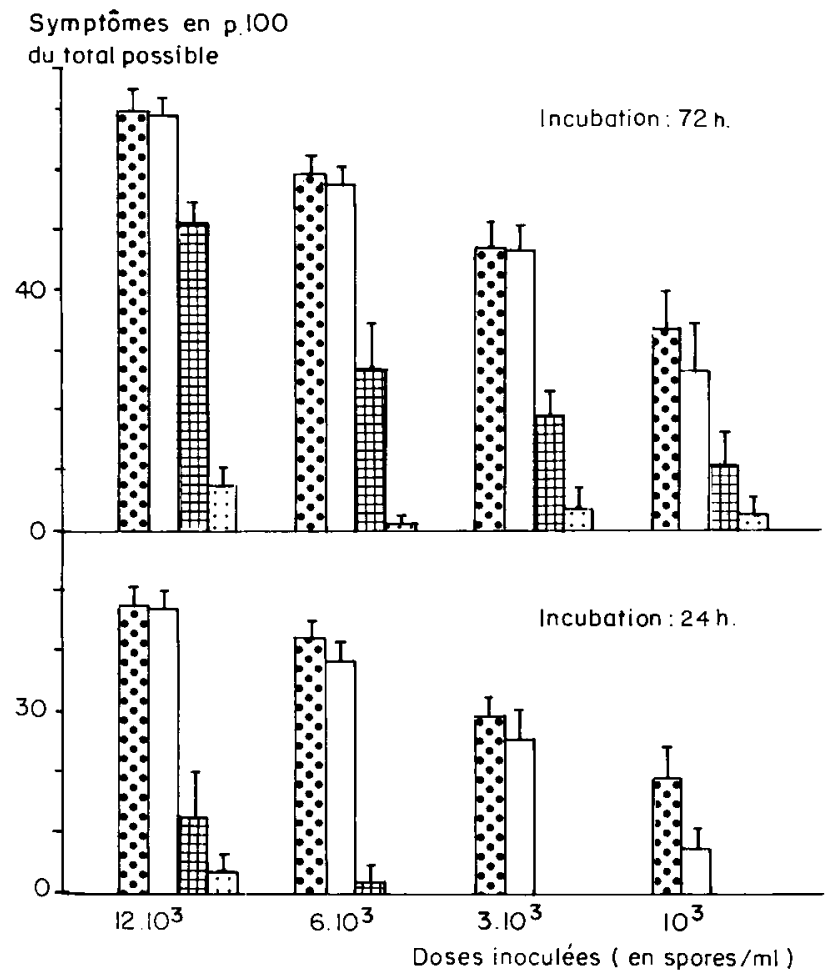

Figure 2

Effets de diverses concentrations de fer (Sulfate ferrique) sur l'expression du pouvoir pathogène de divers inoculums de Botrytis fabae $(A X)$ apportés sur des feuilles détachées de Vicia faba (variété $S 45)$.

$\begin{array}{ll}\because \because \because \mathrm{H}_{2} \mathrm{O} \\ \square & \mathrm{Fe}_{2}\left(\mathrm{SO}_{4}\right)_{3}, 5.10^{-5} \mathrm{M} \\ \square & \mathrm{Fe}_{2}\left(\mathrm{SO}_{4}\right)_{3}, 5.10^{-4} \mathrm{M} \\ \because \therefore \mathrm{Fe} & \mathrm{Fe}_{2}\left(\mathrm{SO}_{4}\right)_{3}, 5.10^{-4} \mathrm{M}\end{array}$

Effect of various iron concentrations (ferric sulfate) on the pathogenic ability of Botrytis fabae $(A X)$ inoculated in a series of doses onto detached leaves of Vicia faba (var. S45).

contre, ils donnent des résultats très différents quant à l'effet sur la croissance des tubes germinatifs (fig. 3). L'EDTA inhibe la croissance surtout pour les spores de B. fabae (AX). Quant au sulfate ferrique, il stimule très significativement la croissance des tubes germinatifs pour les 2 Botrytis testés. Le mélange EDTA + Fer stimule mais à un degré moindre.

Par rapport aux résultats obtenus in vivo, où le fer inhibe le pouvoir pathogène du champignon, nous constatons un contraste remarquable. L'hypothèse d'une action directe du fer sur les conidies des Botrytis paraît donc peu plausible pour expliquer son effet inhibiteur sur les symptômes dus aux Botrytis.

\section{Au niveau de la plante hôte}

Les plantes au stade 4 feuilles bifoliolées sont soigneusement coupées au niveau de la base de la tige. Celle-ci est plongée dans une solution de traitement pendant une durée de $24 \mathrm{~h}$. Ensuite les feuilles sont détachées puis infectées par une suspension de spores de $B$. fabae. D'après les résultats obtenus, il apparaît que les différents traitements n'ont pas modifié la réponse des feuilles à l'infection par $B$. fabae.

Puisqu'il n'apparaît pas de relation directe entre l'apport de fer à la plante et sa résistance au Botrytis, il convient de rechercher une intervention du fer sur un
Longueur des tubes germinatifs en $\mathrm{ym}$ après 24 ?

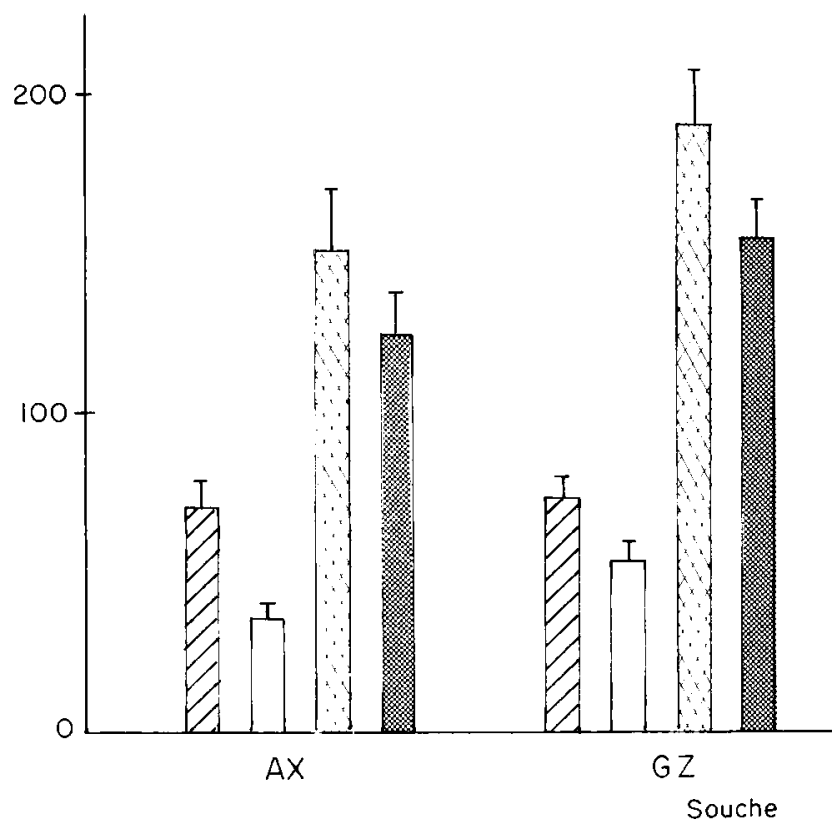

Figure 3

Effets de divers traitements sur la croissance in vitro des tubes germinatifs des spores de Botrytis fabae $(A X)$ et $\mathrm{B}$. cinerea (GZ).

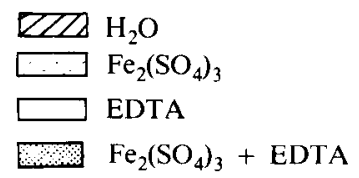

Effects of various treatments on the growth of germ tubes of Botrytis fabae $(A X)$ and $\mathrm{B}$. cinerea (GZ) spores in vitro.

phénomène ayant probablement une conséquence indirecte sur la relation hôte-parasite.

Par ailleurs, la recherche de phytoalexines dans les différents traitements n'a donné que des résultats faibles et inconstants. Nous avons trouvé des zones d'inhibition anti-Cladosporium trop faibles pour pouvoir tenter une identification des différentes phytoalexines de HARGREAVES et al. (1977) et ces zones étaient semblables quel que soit le traitement subi par les feuilles inoculées (sulfate de fer, eau distillée, EDTA, ...). L'absence de phytoalexines dans les gouttes de suspensions de spores et leur très faible présence dans les tissus infectés concordent avec les résultats de HARGREAVES et al. (1977) et de RoSSALl \& MANSFIELD (1980). Ces auteurs expliquent ces observations par la faculté de $B$. fabae de métaboliser activement ces produits antifongiques en les détoxifiant, cette faculté conférant au parasite son pouvoir pathogène sur féverole.

\section{Action de la flore bactérienne du phylloplan}

\section{Présence de deux bactéries}

Après $24 \mathrm{~h}$ d'incubation, 2 populations dominantes de bactéries sont mises en évidence. L'une donne des colonies jaunes très muqueuses produisant une fluorescence jaune-vert sur milieu de KING B. Elle est fortement majoritaire dans les suspensions de spores déposées dans l'eau (tabl. 1). Cette bactérie est en mélange 


\section{TABLEAU 1}

Quantification relative des colonies bactériennes issues des gouttes prélevées après $24 \mathrm{~h}$ d'incubation sur les feuilles.

Relative abundance of bacterial colonies isolated from various conidial drops after $24 \mathrm{~h}$ on leaves.

\begin{tabular}{lcc}
\hline $\begin{array}{l}\text { Origine de } \\
\text { la goutte }\end{array}$ & Aspect des colonies bactériennes \\
\cline { 2 - 3 } & Jaune & Blanche \\
\hline Spores $+\mathrm{H}_{2} \mathrm{O}$ & ++++ & $\varepsilon$ \\
\hline Spores + EDTA & ++ & ++ \\
\hline Spores $+\mathrm{Fe}_{2}\left(\mathrm{SO}_{4}\right)_{3}$ & $\varepsilon$ & ++++ \\
\hline Spores + EDTA $+\mathrm{Fe}_{2}\left(\mathrm{SO}_{4}\right)_{3}$ & ++ & ++ \\
\hline
\end{tabular}

avec une autre bactérie donnant des colonies blanccrème dans les gouttes contenant l'EDT A ou le mélange EDTA $+\mathrm{Fe}_{2}\left(\mathrm{SO}_{4}\right)_{3}$. La bactérie blanc-crème se retrouve pratiquement seule dans le sulfate ferrique.

\section{Identification}

L'identification de ces 2 bactéries a été effectuée. Ce sont des bacilles gram $(-)$. Des galeries API pour bacilles gram (-) ont été réalisées pour chacune d'elles. Les résultats du gram (négatif) et les tests bio-

\section{TABLEAU 2}

Caractères biochimiques différentiels des deux bactéries isolées, selon le système Api pour l'identification des Entérobactéries.

Biochemical characteristics of the two isolated bacteria, according to the Api system for identification of Enterobacteria.

\begin{tabular}{|c|c|c|c|}
\hline & \multirow{2}{*}{ Test } & \multicolumn{2}{|c|}{ Réaction } \\
\hline & & $\begin{array}{l}\text { Bactérie } \\
\text { jaune }\end{array}$ & $\begin{array}{l}\text { Bactérie } \\
\text { blanche }\end{array}$ \\
\hline ONPG & B. galactosidase & - & + \\
\hline $\mathrm{ADH}$ & Arginine $\mathrm{DH}$ & + & - \\
\hline LDC & Lysine DC & - & - \\
\hline ODC & Ornithine DC & - & - \\
\hline CIT & Citrate (Simmons) & + & + \\
\hline $\mathrm{H}_{2} \mathrm{~S}$ & Formation d' $\mathrm{H}_{2} \mathrm{~S}$ & - & - \\
\hline URE & Uréase & - & - \\
\hline TDA & Tryptophane DA & - & - \\
\hline IND & Indole & - & - \\
\hline VP & Acétoine & - & + \\
\hline GEL & Gélatine & + & - \\
\hline GLU & Glucose & + & + \\
\hline MAN & Mannitol & - & + \\
\hline INO & Inositol & - & - \\
\hline SOR & Sorbitol & - & - \\
\hline RHA & Rhamnose & - & - \\
\hline SAC & Saccharose & - & - \\
\hline MEL & Melibiose & + & - \\
\hline $\mathrm{AMY}$ & Amygdaline & - & + \\
\hline ARA & Arabinose & + & - \\
\hline $\mathrm{OX}$ & Oxydase & + & - \\
\hline NIT & Nitrites & - & + \\
\hline
\end{tabular}

chimiques API pour identification des Entero-bactéries (tabl. 2) nous incitent à penser que la bactérie à colonies jaunes est un Pseudomonas fluorescens Migula, alors que celle donnant des colonies blanc-crème s'apparente à un Erwinia (probablement E. herbicola (Löhn.) Dye $=$ Enterobacter agglomerans). Pour cette dernière cependant, il convient de noter quelques anomalies par rapport aux résultats de DYE (1969). Nous nous sommes conformés au tableau de différenciation des Entérobactériacées fourni par API-System et dans lequel ces mêmes anomalies sont observées concernant $E$. agglomerans notamment $\mathrm{H}_{2} \mathrm{~S}$ négatif $(100 \mathrm{p} .100)$, gélatine négatif (98 p. 100), arabinose négatif (6 p. 100) et rhamnose négatif $(21 \mathrm{p} .100)$

\section{Antagonisme Pseudomonas - Erwinia in vitro}

Les premiers résultats acquis suggèrent la présence d'une activité antagoniste chez Pseudomonas vis-à-vis d'Erwinia, activité inhibée par addition de sulfate ferrique.

Cette antibiose a été éprouvée en boîte de Petri sur milieu de KING B. On dépose le Pseudomonas au centre de la boîte $\left(10 \mu \mathrm{l} / 10^{8}\right.$ germes $\left.\mathrm{ml}\right)$. Après $24 \mathrm{~h}$ d'incubation à $30^{\circ} \mathrm{C}$, les colonies sont tuées au chloroforme puis éliminées. On pulvérise alors une suspension d'Erwinia $\left(10^{8}\right.$ germes $\left./ \mathrm{ml}\right) .24 \mathrm{~h}$ après la pulvérisation, des zones d'inhibition sont visibles dans la culture d'Erwinia à l'endroit de la culture de Pseudomonas et largement autour de ce périmètre. La même expérience dans un milieu contenant du sulfate ferrique $\left(10^{-4} \mathrm{M}\right)$ ne permet pas d'observer d'antibiose. Dans un test semblable, Erwinia n'exerce pas d'inhibition sur la croissance de Pseudomonas.

\section{Action des deux bactéries vis-à-vis du champignon parasite}

\section{a) Action « in vitro » sur la germination et la} croissance

Ces bactéries purifiées ont été éprouvées in vitro pour déterminer leur action sur les conidies et sur le mycélium de $B$. fabae.

- Effet sur la germination des conidies.

L'action des bactéries a été éprouvée sur la germination des conidies (tabl. 3) et sur la croissance des tubes germinatifs, dans l'eau et dans du sulfate ferrique (fig. 4).

Pseudomonas n'exerce pas d'effet significatif sur ces 2 paramètres. Par contre, Erwinia inhibe la germination des spores et la croissance des tubes germinatifs dans l'eau mais également la croissance des tubes germinatifs dans du sulfate ferrique. Erwinia impose son action inhibitrice sur l'allongement des tubes germinatifs lorsque les 2 bactéries sont utilisées en mélange dans l'eau comme dans le sulfate ferrique, mais il semble que l'effet soit obtenu par la simple densité d'inoculum et non par une multiplication bactérienne dans ce milieu où les seuls nutriments sont apportés par les conidies du champignon en germination.

- Effet sur la croissance mycélienne en boîte de Petri.

Une suspension bactérienne d'Erwinia disposée dans des puits inhibe la croissance mycélienne de $B$. fabae (le 
TABLEAU 3

Effet des deux bactéries isolées (Pseudomonas $=P_{S} ;$ Erwinia $\left.=E r\right)$ du phylloplan sur la germination des conidies de Botrytis fabae $(A X)$ in vitro. Effect of two bacteria from leaves of Vicia faba on germination of conidia of Botrytis fabae $(A X)$ in vitro.

\begin{tabular}{|c|c|c|c|c|c|c|c|c|}
\hline Germination testée dans & \multicolumn{4}{|c|}{$\mathrm{H}_{2} \mathrm{O}$} & \multicolumn{4}{|c|}{$\mathrm{Fe}_{2}\left(\mathrm{SO}_{4}\right)_{3}$} \\
\hline Traitement & $\mathrm{H}_{2} \mathrm{O}$ & Ps & Er & $\mathrm{Ps}+\mathrm{Er}$ & $\mathrm{H}_{2} \mathrm{O}$ & Ps & Er & $\mathrm{Ps}+\mathrm{Er}$ \\
\hline Pourcentage de germination & 95,1 & 96,2 & 81,95 & 81,9 & 95 & 88 & 89 & 91,4 \\
\hline $\begin{array}{l}\text { Intervalle de confiance (avec un coeffi- } \\
\text { cient de sécurité de } 95 \text { p. 100) }\end{array}$ & 2,4 & 2,8 & 6,7 & 6,2 & 2,8 & 5,8 & 3,5 & 5,2 \\
\hline
\end{tabular}

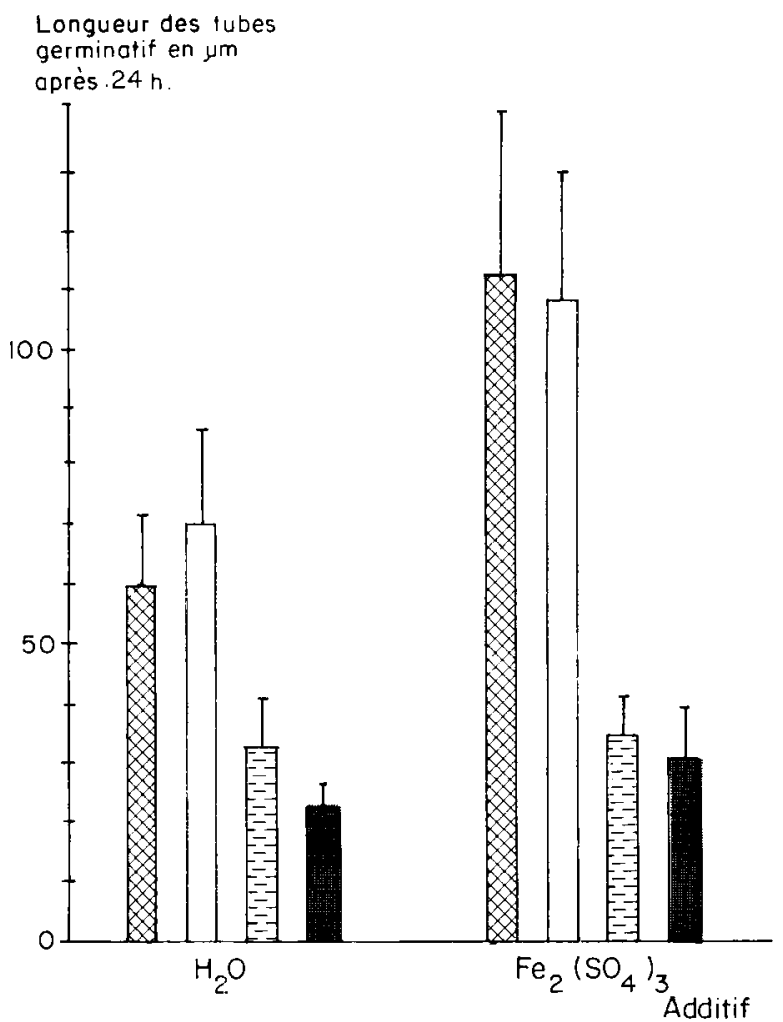

$\mathrm{H}_{2} \mathrm{O}$ $E=-7$ Erwinia

Pseudomonas

Pseudomonas + Erwinia

Figure 4

Effets de deux bactéries du phylloplan de Vicia faba sur la croissance des tubes germinatifs des spores de Botrytis fabae $(A X)$ in vitro dans l'eau ou le sulfate de fer.

Effects of two bacteria from the leaf surface of Vicia faba on the growth of germ tubes of Botrytis fabae $(A X)$ spores in vitro in water or ferric sulfate.

mycélium contournant les puits) alors que, dans le cas d'une suspension de Pseudomonas, le mycélium vient au contact même des puits bactériens. Pseudomonas n'a donc aucun effet apparent dans ces conditions expérimentales.

b) Action sur l'expression du pouvoir pathogène in vivo

Les 2 bactéries isolées des feuilles de $V$. faba ont été éprouvées quant à leur effet sur l'expression du pouvoir pathogène de $B$. fabae, sur feuilles détachées selon le protocole habituel, soit en présence de sulfate ferrique, soit en présence d'eau distillée.

Dans l'eau distillée, l'apport d'Erwinia ne modifie pas le niveau des symptômes par rapport au témoin (fig. 5). Ce résultat peut s'expliquer par la présence naturelle de Pseudomonas à la surface des feuilles, qui

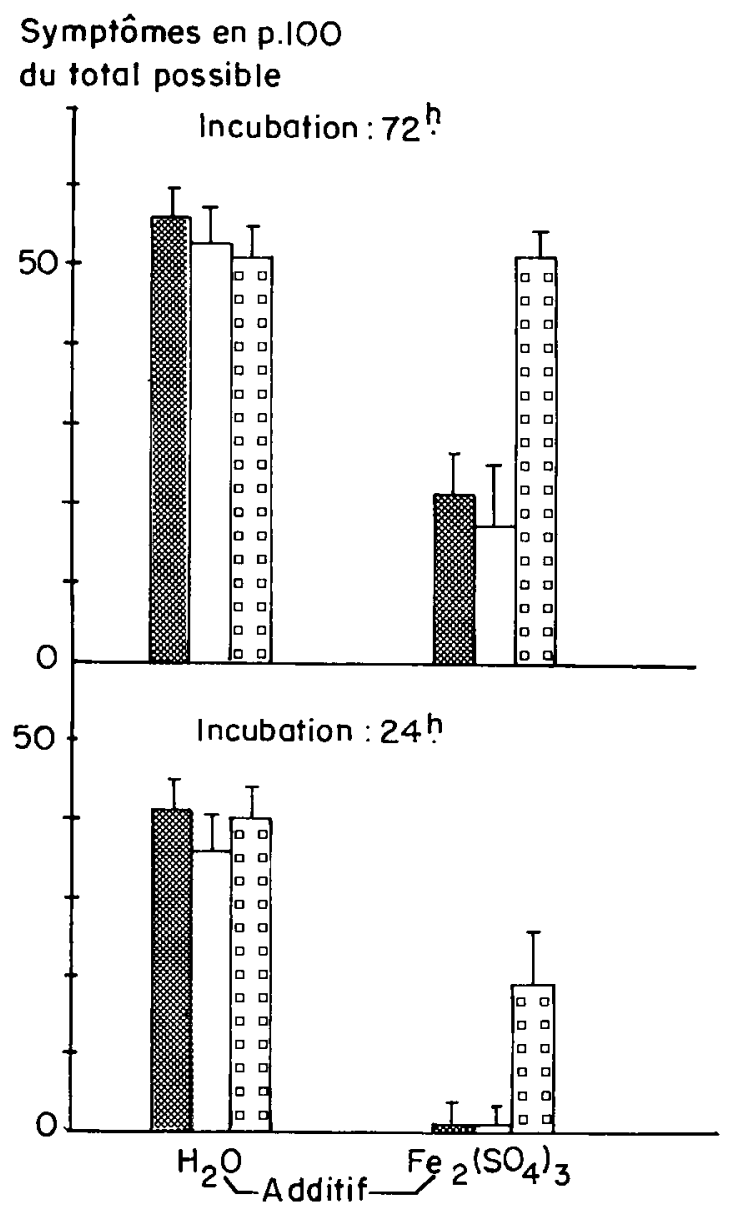

Figure 5

Modulation par les deux bactéries isolées du phylloplan de Vicia faba, de l'inhibition de Botrytis fabae $(A X)$ par le sulfate ferrique sur feuilles détachées de Vicia faba.

$\mathrm{H}_{2} \mathrm{O}$

Erwinia

區品 Pseudomonas

Souche AX

variété S45

Effect of two phylloplane bacteria on the inhibition of Botrytis fabae $(A X)$ by ferric sulfate on detached leaves of Vicia faba. 
interdirait l'installation et la multiplication d'Erwinia arrivant dans un milieu déjà colonisé où le fer a été mobilisé par les premiers occupants.

Par contre, en présence de sulfate ferrique, l'inhibition des symptômes, très forte avec Erwinia, est partiellement annulée avec Pseudomonas (surtout après 72 h d'incubation).

Ces derniers résultats confirment l'effet inhibiteur d'Erwinia mais suggèrent, pour Pseudomonas, la présence d'une activité favorable à $B$. fabae puisqu'en présence de cette bactérie, l'inhibition due au sulfate ferrique est pratiquement supprimée.

Une étude des activités présentes dans les filtrats de cultures bactériens est en cours afin de préciser les observations faites précédemment.

\section{DISCUSSION - CONCLUSION}

Le sulfate ferrique utilisé à doses faibles $\left(10^{-4} \mathrm{M}\right)$ a divers effets sur l'interaction Botrytis - féverole ;

- Il inhibe l'apparition des taches nécrotiques dues aux Botrytis sur feuilles détachées de $V$. faba.

- Il stimule la germination et la croissance des tubes germinatifs de $B$. fabae in vitro.

- Il modifie la microflore bactérienne du phylloplan de $V$. faba en faisant disparaître $P$. fluorescens au profit d'Erwinia.

En outre, Erwinia inhibe in vitro la germination et la croissance des tubes germinatifs de $B$. fabae alors que $P$. fluorescens, ajouté à la goutte d'inoculum sur feuilles détachées, lève l'inhibition du sulfate ferrique sur l'apparition des taches nécrotiques des Botrytis.

Enfin, Pseudomonas montre, in vitro, un antagonisme vis-à-vis d'Erwinia, antagonisme levé par une culture en présence de fer $\left(10^{-4} \mathrm{M}\right)$.

Contrairement aux résultats de BROWN \& SWINBURNE (1982) concernant $B$. cinerea sur feuilles de féverole, l'EDTA ne stimule pas l'agressivité de $B$. fabae dans nos conditions expérimentales, alors que le sulfate ferrique l'inhibe fortement. Cependant, la principale différence entre les 2 expérimentations est liée à la forte agressivité des souches que nous utilisons lors d'infections normales à l'aide de suspensions de spores dans l'eau. De même, ces souches germent à plus de $95 \mathrm{p}$. 100 dans l'eau distillée, alors que celles de $B$. cinerea, utilisées par BROWN \& SWINBURNE (1982) ou celles de C. musae (BROWN \& SWINBURNE, 1981) et de Glomerella cingulata (ADIKARAM et al., 1982) qui montrent des réponses identiques vis-à-vis des chélateurs du fer, semblent dépendre d'additifs nutritionnels à la fois pour leur germination et leur pouvoir pathogène.

Toutefois, le fer constitue un puissant stimulant de la croissance des tubes germinatifs, même pour les souches que nous utilisons.

Il paraît donc anormal d'observer une inhibition très importante du développement des nécroses lorsque les suspensions de spores sont utilisées en présence de fer. Cette inhibition pourrait être expliquée par une stimulation de la résistance de la plante. Il semble que ce ne soit pas le cas puisque l'administration du fer par le système vasculaire ne modifie en rien la sensibilité et que, par ailleurs, la concentration en phytoalexines dans les premières heures de l'interaction reste faible ou nulle et identique pour tous les traitements.

Par contre, les modifications de microflores bactériennes dans les gouttes de suspensions d'inoculum expliquent les réponses radicalement différentes en présence ou en absence de fer. Les bactéries isolées pourraient posséder des caractères concordant avec les résultats observés. C'est ainsi qu'E. herbicola semble produire une activité antibiotique susceptible d'inhiber la croissance des Botrytis. Quant à $P$. fluorescens, la régulation de ses populations dans le phylloplan par l'ion ferrique semble prouver qu'il appartient bien au groupe de $P$. fluorescens producteur de sidérophores (TEINTZE et al., 1981).

Des modifications de microflore du phylloplan liées à une modulation de l'expression des symptômes sur feuilles détachées ont déjà été signalées sur féverole (RoSSALL \& MANSFIELD, 1980). Nous ajoutons à ces observations une explication au moins partielle de l'action des bactéries par la production de sidérophores favorisant l'expression du pouvoir pathogène des Botrytis. Une explication de cette nature a été proposée pour rendre compte de l'effet de Pseudomonas sp. sur la germination (Mc CRACKEN \& SWINBURNE, 1979) et sur le pouvoir pathogène des conidies de $C$. musae sur banane (BROWN \& SWINBURNE, 1981).

L'ion ferrique interviendrait sur les populations de Pseudomonas en réprimant, par sa présence, la production des sidérophores (Mc CRACKEN \& SWINBURNE, 1979 ; KLOEPPER et al., 1980), ce qui ferait perdre à ces bactéries l'avantage sélectif dont elles bénéficient dans un milieu pauvre en fer.

Par ailleurs, nous avons vu qu'au moins certaines souches d'E. herbicola sont antagonistes des Botrytis et leur multiplication, accompagnée de la diffusion d'un produit antifongique, pourrait expliquer une autre partie de la diminution de l'agressivité du champignon.

D'un point de vue pratique, les résultats ouvrent d'intéressantes perspectives pour essayer de limiter l'extension des dégâts dus aux Botrytis sur féverole d'hiver en zone atlantique. Il est possible d'envisager l'effet de traitements préventifs au sulfate de fer, au début de la floraison sur le développement ultérieur de l'épidémie. Par ailleurs, une étude plus approfondie de l'activité et de la nature de la substance antifongique produite par $E$. herbicola est à envisager.

D'un point de vue plus fondamental, il conviendra aussi d'analyser la flore microbienne du phylloplan d'une collection étendue de variétés de féverole, dans le but d'y découvrir éventuellement des différences que l'on pourrait relier à la constitution génétique.

Enfin, il serait bon d'envisager une amélioration de l'efficacité protectrice d'E. herbicola ainsi qu'une adaptation à d'autres plantes comme la vigne qui subit de graves dégâts de $B$. cinerea. Dans le cas d'un support plasmidial de la production du sidérophore de $P$. fluorescens - ce qui a déjà été observé pour d'autres sidérophores microbiens (CROSA, 1980 ; GRIFFITHS et al., 1980 ) - un transfert de cette information vers $E$. herbicola pourrait constituer pour celui-ci un avantage sélectif vis-à-vis de la microflore du phylloplan. 


\section{RÉFÉRENCES BIBLIOGRAPHIQUES}

\begin{abstract}
Abou-Zeid N., 1978. Contribution à la connaissance de la résistance naturelle de Vicia faba contre les Botrytis. Thèse Doct. Ingén. ENSA de Rennes, Univers. Rennes 1.154 p.
\end{abstract}

Adikaram N. K. B., Brown A. E., Swinburne T. R., 1982. Rotting of immature Capsicum frutescens L. fruit by iron-depleted Glomerella cingulata Stonem. Physiol. Plant Pathol., 21, 171-177.

Brown A. E., Swinburne T. R., 1981. Influence of iron and iron chelators on formation of progressive lesions by Colletotrichum musae on banana fruits. Trans Br. Mycol. Soc., 77, (1), 119-124.

Brown A. E., Swinburne T. R., 1982. Iron-chelating agents and lesion development by Botrytis cinerea on leaves of Vicia faba. Physiol. Plant Pathol., 21, 13-21.

Crosa J. H., 1980. A plasmid associated with virulence in the marine fish pathogen Vibrio anguillarum specifies an iron-sequestering system. Nature, 284, 566-568.

Dye D. W., 1969. A taxonomic study of the genus Erwinia. III. The herbicola group. N. Z. J. Sci., 12, 223-236.

Griffiths E., Rogers H. J., Bullen J. J., 1980. Iron, plasmids and infection. Nature, 284, 508-509.

Hargreaves J. A., Mansfield J. W., Rossal S., 1977. Changes in phytoalexin concentrations in tissues of the broad bean plant (Vicia faba L.) following inoculation with species of Botrytis. Physiol. Plant Pathol., 11, 227-242.

Keen N. T., 1978. Phytoalexins : efficient extraction from leaves by a facilitated diffusion technique. Phytopathology, 68, 1237-1239.

King E. O., Ward M. K., Raney D. E., 1954. Two simple media for the demonstration of pyocyanin and fluorescin. J. Labor. Clinic. Med., 44, 301-307.
Klement Z., 1965. Method of obtaining fluid from the intercellular spaces of foliage and the fluid's merit as substrate for phytobacterial pathogen. Phytopathology, 55, 1033-1034.

Kloepper J. W., Leong J., Teintze M., Schroth M. N., 1980. Enhanced plant growth by siderophores produced by plant growth promoting rhizobacteria. Nature, 286, 885-886.

Lankford C. E., 1973. Bacterial assimilation of iron - CRC Crit. Rev. Microbiol., 2, 273-331.

Leach R., Moore K. G., 1966. Sporulation of Botrytis fabae on agar cultures. Trans. Br. Mycol. Soc., 49 (4), 593-601.

Mc Cracken A. R., Swinburne T. R., 1979. Siderophores produced by saprophytic bacteria as stimulants of germination of conidia of Colletotrichum musae. Physiol. Plant Pathol., 15, 331-340.

Mansfield J. W., Deverall B. J., 1974. The rates of fungal development and lesion formation in leaves of Vicia faba during infection by Botrytis cinerea and B. fabae. Ann. Appl. Biol., 76, 77-89.

Rossall S., Mansfield J. W., 1980. Investigation of the causes of poor germination of Botrytis spp. on broad bean leaves (Vicia faba L.) Physiol. Plant Pathol., 16, 369-382.

Schroth M. N., Hancock J. G., 1982. Disease suppressive soil and root-colonizing bacteria. Science, 216, 1376-1381.

Teintze M., Hossain M. B., Baines C. L., Leong J., Van der Helm D., 1981. Structure of ferric pseudobactin, a siderophore from a plant growth promoting Pseudomonas. Biochemistry, 20, 6446-6457.

Weinberg E. D., 1966. Roles of metallic ions in host-parasite interactions. Bacteriol. Rev., 30 (1), 136-151.

Weinberg E. D., 1978. Iron and infection. Microbiol. Rev., 42 (1), 45-66. 